\title{
On the Design of User Experience Based Persuasive Systems
}

\author{
Ahmad Jawdat (Corresponding author) \\ College of Arts and Sciences, University of Nizwa \\ P.O. Box 33, Nizwa 616, Oman \\ Tel: 96-82-544-6543Ｅ-mail: jawdat@unizwa.edu.om \\ Qasem Obeidat \\ College of Arts and Sciences, University of Nizwa \\ P.O. Box 33, Nizwa 616, Oman \\ Tel: 96-82-544-6543E-mail: qasem@unizwa.edu.om \\ Alaa Aljanaby \\ College of Arts and Sciences, University of Nizwa \\ P.O. Box 33, Nizwa 616, Oman \\ Tel: 96-82-544-6297_E-mail: alaa@unizwa.edu.om
}

Received: May 6, 2011

Accepted: June 1, 2011

doi:10.5539/cis.v4n4p90

\begin{abstract}
The objective of persuasive technology researches is to develop persuasive systems that are able to change or reshape human behavior. Persuasive technology has quickly found a wide range of applications in many fields of research and development like marketing, health, safety and environment. The key element in designing successful persuasive systems is the improvement of the persuasion process. An important factor that should be included in the persuasion process is the user experience. This paper reviews the current trends of persuasive technology and shows some example of the available persuasive systems. The contribution of this paper is proposing a new and promising research direction for building persuasive systems that take the user feedback as a key element in the persuasion process. Some of the systems that follow this approach have been proposed and illustrated.
\end{abstract}

Keywords: Persuasive Technology, Persuasive Systems, User Experience, Web and Mobile Persuasive, Ambient Persuasive, E-commerce, Web Advertising

\section{Introduction}

Fogg $(1998,1999)$ has defined persuasive technology as the use of computing technology to change or reshape the attitude or behavior of the user. Persuasive technology is recently used in many applications such as web-based applications, portable devices, stand-alone programs, computerized toys, games and robotics. However, there are many other application areas that are candidate to use persuasive technology such as politics, religion, health, safety, self-improvement and environment protection (Cheng, 2003). The persuasive technology can be classified as a tool, media or social actors' based on the functional role of the computing device (Fogg, 1999, 2003). As a tool, persuasive technologies increase the ability of users to perform a target behavior by simplifying the target. However as a media, persuasive technologies can allow people to experience information developed to sharp their opinions and as actors it can take on pseudo-human roles.

A model for the human behavior was proposed by Fogg (2009) called Fogg Behavior Model (FBM). The model was to make a better understanding of elements involved in human behavior and thus help in developing more efficient persuasive systems. Motivation, ability and triggers are the three factors that were included in FBM. In order for a human to perform a target behavior, based on FBM, he should be motivated enough, has the ability and need to be triggered to do so. Examples of motivators are pleasure/pain, hope/fear and acceptance/rejection. And examples for the ability factor are time, money and physical effort while the triggers examples are spark, 
facilitator and signal. However, the persuasion process can also make an advantage from the accumulated experiences of previous users who used some system and put their feedback. The user feedback can play a main role in persuading future users to do or not to do the targeting behavior.

This paper is proposing the approach of designing persuasive systems based on users' experiences as an attempt to develop more effective persuasive systems. The rest of this paper is organized as follows. Some research domains of persuasive technology are first discussed in Section 2. Section 3 briefly surveys some recently developed persuasive systems. The new approach of using user experience as a key factor in developing persuasive systems is then discussed in Section 4. The conclusion of the paper is given in Section 5.

\section{Persuasive Technology Research Domains}

Persuasive technology has been used in many research domains. Ambient, games, web, and mobile are some examples of these domains. These domains required huge research efforts in the past few years and they are briefly reviewed in the next subsections.

\subsection{Ambient Persuasive}

The term ambient persuasive is part of a larger domain which is the Ambient Intelligence (AmI) which refers to embedding intelligent systems into the environment where they interact with users everywhere and anytime during their daily lives. Ambient Intelligence is seen as the next step for Artificial Intelligence (AI). The AI techniques and methods are very useful in developing AmI systems (Ramos, Augusto, \& Shapiro, 2008). Embedding persuasive systems within environment is called ambient persuasive or environmental persuasion (Mathew, 2005). Ambient persuasive systems are mainly installed in public places to make the best benefit from using them. Moreover, using ambient persuasive allows people to be persuaded, without being annoyed, in the right time and at the right place. This means that they will gain the most benefit from persuasion, especially if the message that will be delivered is simple and direct (Intille, 2004). Ambient persuasive systems may be found in anyplace in home (e.g. energy consumption), factory (e.g. motivation and productivity), shopping malls (e.g. advertising or specific consumer behaviors), office, classroom, vehicle, hospital, etc.

A vehicle is a popular place where you can think of attaching a persuasive system. These systems targeted some behaviors like the driver speeding or attaching safety belt for more safety by alerting the driver using sound or light (Oinas-Kukkonen \& Harjumaa, 2008). Many complex systems have suggested different approaches to ensure better economic driving behavior. EcoMatic system (Meschtscherjakov et al., 2009) automatically reduces the fuel consumption when it is activated by the driver and at the end of the trip it shows the amount of saved fuel. It works by adjusting various parameters within the car such as changing the engine into an idle mode when the car stops moving. Another system that is used in vehicles is CommuterNews (Tester, Fogg, \& Maile, 2000) which aimed to motivate the driver to listen to the news by providing questions and making competition.

WaterBot (Arroyo, Bonanni, \& Selker, 2005) is another clear example of ambient persuasive, where the information appear on a device that is attached to the sink itself; the information displayed are used to motivate people to turn off the tap when the water is not being used which will reduce the amount of wasted water. It relies on sound and light to motivate water savings. The CleanSink (Arroyo, Bonanni, \& Selker, 2005) system was also suggested adding a small display beside the sink that can persuade people to wash their hands properly by following the directions displayed. It was suggested to be used in hospitals, restaurants, and industrial clean rooms. In hospitals' examination rooms, CleanSink can control the room lights, so if the staff washes their hands the lights will turn on.

\subsection{Web and Mobile Persuasive}

Most of the persuasive systems available were developed as web sites, mobile systems, games or embedded systems within the environment. Using Web based systems, and mobile applications can give a great opportunity to spread persuasive systems and to interact with users, where they can be reached easily. One of the persuasive websites is the www.scorecard.org website; this website helps users to participate in protecting the environment (King \& Tester, 1999). There are many studies available on Human Computer Interaction that discussed persuasion of the interface itself. Those studies concentrated on methods to make the user trust a website and to take action rather than surf only, and to persuade him to open the website regularly. Persuasion and promotion are the key success for any new website.

A mobile phone application that alerts a person to call friends periodically (Marcus \& Chen, 2002) is an example of using persuasive technology in mobiles. Another mobile device application called SmartTrip (. Johnson et al., 2007) aims to combine multiple trips into a single trip in order to reduce driving. Also an environmental sustainability related application is the GreenScanner (Tomlinson, 2008) which is a mobile application that can 
be used at stores to give detailed information about the environmental impacts of various products using their barcodes. Another mobile application introduced in (Intille et al., 2003) helps people to select healthy food by providing comparisons between two or more products on one aspect of dietary that the customer determines which gives simple information for healthier food.

\subsection{Game Persuasive}

During the last few years, games have been founded as a common and an important form of persuasive technology. Each game must have advantages benefiting the players into different domain like health, education, religion, culture, business, etc. It is better if the game has a web-based game interface to reach a large number of people with a low cost (Khaled et al., 2006). Using games as a persuasive tool helps game players to change their behaviors by supplying them with effective knowledge during their play.

Some games are controlled by the player's body movements like Nintendo Wii or PlayStation Move or Xbox360 Kinect. A player using Wii for example can play tennis by holding a stick and moving his hand and body as in the reality. Another game is Konami's Dance Dance Revolution (DDR) which is revolves around the concept of dancing. The dancer here moves on different places on a floor pad to match the screen display marking. These games and others can be viewed as persuasive systems as they encourage players to be active. (Khaled, 2008).

The SuperKids game Drop \& Catch is an educational computer game designed by Dole Food Company. This game teaches children the advantages of eating 5 to 9 different kinds of fruits and vegetables per a day. The kid player Bam-Nana should pack super food snacks to give him the boost he will need. The super kid friend Brocco is giving him a hand tossing nutritious snacks as fast as he can. But his enemy Sat Fat wants to sabotage things with junk foods. Bam-Nana wins if he catches 5 super foods respectively; if he catches one item of junk food he must start from the beginning and remove all the super foods that he collected before, refer to www.dole.com.

\section{Persuasive Systems}

Many persuasive systems have been developed since the early work of Fogg $(1998,1999,2003)$. The following subsections briefly surrey the most interesting available persuasive systems.

\subsection{Recommender Systems}

Recommender systems are considered as collaborative filtering systems (Herlocker, Konstan, \& Riedl, 2000). They use people opinions and data to build recommendations for other users who may share the same interests. Nowadays, they are used by many e-commerce websites. Researches in this area proved that users showed interest in these kinds of systems. Researchers now are investigating how to improve the value of recommendations and make them more persuasive for the users to make more benefit from them (Konstan, 2004).

One of the good methods suggested for building recommendations depend on making groups of people who have a similar profile of interests. The profile of interests is created by collecting data about users when they bid on, rate or buy a specific product. The system then uses these similar profiles to build recommendations (Cheng, 2003). Recommender systems are very important in businesses especially in e-commerce. They can increase sales and help people find interesting items. One good application is the MovieLens, it is a web site for recommending movies where you can rate movies you already seen and depending on your ratings the system will suggest movies to watch that match your taste (Cosley et al., 2003).

\subsection{Decision and Consumer Support Systems}

Decision support systems help decision makers and support them in the decision making process, these systems mostly refer to people in high management positions. Some other references introduced them as consumer decision support systems which help online users make purchasing decisions in e-commerce web sites (Zhang, \& $\mathrm{Pu}, 2006)$. The reliance on decision support systems is optional, that's why it depends on how persuasive they are. The persuasiveness of these systems will convince the decision maker to rely on the decision support provided. There are many factors that affect the overall persuasiveness of these systems, the complexity of the decision, the person using the system and the message delivered by the system (Alison, 2009). An example is a system that assists consumers to reduce the number of unnecessary visits to health care facilities.

\subsection{Self Monitoring Systems}

These systems allow people to monitor themselves in order to modify their behaviors to achieve a predetermined goal (Fogg, 2003). This type of persuasive systems help people continuing the behavior change process rather than convincing them to start changing the undesired behavior. For example UbiFit (Consolvo, Landay, \& McDonald, 2009) is an interactive application that helps people monitors their daily physical activities. They can 
define a weekly target and view progress toward their predefined goal. UbiFit uses a display image for a garden with flowers that resides on the background screen of a mobile phone. The garden represents one week's activities and the flowers of different colors represent different types of activities. This image could be changed over time because it may get boring.

\subsection{Simulation and Virtual Reality Systems}

Simulated environment is similar to the real ones; they can persuade people to change their behaviors by obtaining some experiences and observe the link between cause and effect (Fogg, 2003). People will make decisions based on the experiences they gain from using these systems. Virtual reality is also a powerful simulation tool that has a great effect in persuading people (Chittaro \& Zangrando, 2010). A good example of persuasive simulation is a computerized doll called "Baby Think It Over", used to motivate responsible sexual behavior. Another example that used virtual reality was presented by Chittaro and Zangrando (2010) to simulate the effects of human distress on attitudes towards fire safety. It simulates the experience of evacuation of a burning building and the effects of staying in smoke or avoiding it.

\subsection{Dialogue and Negotiation Systems}

Persuasive dialogue systems can be used to affect and change people attitudes or behaviors by sending advice and argument persuasive messages to them (Nguyen \& Masthoff, 2010). There is a positive correlation between acceptance of an argument and change in beliefs and attitudes but acceptance does not guarantee changes. For example, smokers who received and accepted a persuasive message which highlights the smoking problem about their health, but they are still smoking and did not change their attitude (Nguyen \& Masthoff, 2010). Persuasion through dialogue is novel fields of Human Computer Interaction. Some of the important researches have applied persuasive communication principles to natural language generation (Andrews, Manandhar, \& De Boni, 2008). The Daphne system is an example of such researches which aimed to improve people's diet by using arguments. The researchers who developed the Daphne system believe that changing opinions of an individual is a conflict situation between two parties, and this conflict can be resolved using direct advice through the use of dialectic argumentation (Grasso, Cawsey, Jones, 2000).

\subsection{Data Visualization Systems}

Data visualization systems provide variant displays that are able to deliver information to users in a pleasant way. Its target is to modify human behavior by increasing their awareness (Vande-Moere, 2007). As an example; DiMicco, Pandolfo, and Bendern(2004) used a graphical display that shows the rate of participation of each group member in a conversation. He studied the effects of this display in convincing members who had low participation rates to participate more and vice versa. Another example is social-network visualizations that are used to promote awareness of social health for elderly people. The visualizations intended to motivate social engagement among older adults to help them become more aware of their ability to participate in interactions (Morris, 2005).

\section{User Experience Based Persuasive Systems}

The persuasive technology is still in its beginning and there are so many ideas that could be implemented in a lot of fields. Persuasive applications can cover various domains, and many of them still need to be discovered and developed. In this section we will introduce some of our innovative ideas to share with the fellow researchers of this technology. To start with education for example; many ideas could be implemented to persuade people in order to read more, educate themselves, or encourage them to join a college. A mobile application that introduces information in a form of questions and answers, along with the use of ranking to motivate users to gain the knowledge is one example. Another important domain is politics; political parties may add an automatic negotiating agent in the web to convince people in order to join their party. These kinds of systems and many more use artificial intelligence techniques such as natural language processing and machine learning.

Furthermore, there are many other challenging applications that could be used to convince people to do or stop doing behaviors such as: drugs, alcohol and internet addiction. In addition, they could be used to promote healthy lifestyle activities like practicing, or to persuade employees to put more effort and time in their work. On the other side, applications could be developed to convince terrorists to stop bombing, or to influence someone with specific religious thoughts or ideas.

We suggest introducing experience based persuasive systems, refer to Figure 1, which depend on other people personal experiences. The system automatically generates useful information based on the acquired experiences to help others in decision making. By using this idea we can make benefits from the knowledge of many people all over the world about a specific action or behavior. We believe that involving users in the persuasion process 
would give more credibility to the system. The system will automatically analyze the collected data, and compute statistics to create suggestions and recommendations. As a result these recommendations are used to convince other users to follow or to stop a specific action. Numerous ideas could be given as examples for the use of this method, and we will mention three.

\subsection{E-Commerce and Customers Persuading Process}

People frequently seek professional advice before buying a certain product. They would usually ask a friend or a salesman or simply surf the web for the required information. In fact most of us need to be convinced about a specific product before buying it. This is one of the most important drawbacks of E-commerce comparing with the traditional trading process. With the use of persuasive system, we might reduce the effect of this factor. Using other people experience feedbacks when buying a product will increase the confidence of customer and ease the decision making on him.

People proved through the experience of wikis and forums that they tend to share their knowledge with others. In e-commerce the lack of an appropriate platform is a major obstacle that prevents users from participating and sharing their own experiences. For internet customers, seeing statistics and information about a specific product is relieving before buying or participating in an auction because the product is intangible. The system should collect data about products from people who had used it; this will give more credibility for new customers to trust the system's output. Statistics and charts can be generated by the system and introduced in a simple and readable way to customers, in order to convince them to buy the product.

Figure 2 shows an example of how the user's experiences can be viewed as a column chart so it is easy to read by any customer. It shows two colors; the green indicates good feedback while the red indicates drawbacks. At the end there is a total satisfaction column showing the degree of users' contentment. On the title you can see the number of users who shared their experience while using this specific mobile phone, more users give more credibility and accuracy to the calculated statistics.

From the website owner's view, the persuasive system will help to increase the sales and will also provide feedback about the products for manufacturer's pro future development. This data in the system is valuable, since it reflects the degree of satisfaction of the customers about the products from real life use. Thus, the information is very important for manufacturers to induce the decision makers to enhance their production in general. They could change their materials or pay more for research groups or even change the whole product into a new improved version.

Another part that could be added to the persuasive system is a price reasonability system. This system should be embedded within e-auction web sites. In auctions one of the customers' main concerns is that when should they stop bidding and whether the deal they won is a bargain or not. It is quite often that the customer might be interested in a specific item, and has no idea how much it is worth, especially used items. The pricing reasonability system helps customers to know whether the price of the item is reasonable or not by giving a green color or red for the auction price. The system then can automatically calculate this value by comparing similar items already sold with the opportunity of showing details of those items.

Moreover, the system could generate the collected users' experiences and display it in the form of suggestions for customers who didn't decide exactly which model of the product they desire to buy yet. For example, if a customer wants to buy a mobile phone, he can provide the system with his own requirements and expectations (Figure 3). The system then can produce a list of suggestions (refer to Table 1) to the customer, sorted by the best results given by other users. In the example below, the user specified some requirements in the first column which can be used to narrow the list of suggested items. The data in the second column is calculated using users' experiences from using the same phone. The results will appear as shown in Table 1.

\subsection{Persuading Businesses to Participate in Web Advertising}

Many of the available websites today depend on advertisement as a major source of their revenue model. The well known websites usually do not face major problems in attracting businesses to advertise using there websites. On the other hand, websites with a limited number of users may struggle to convince business decision makers to use their websites for advertising. The proposed system will benefit both types of websites to increase their total daily income from advertisements, using automated persuasive system to draw advertisers. Additionally, many of the small and medium businesses need to be convinced to spend more money on advertising. They need to be encouraged to spend more money on advertising by showing them that it will support their business and help them gain more profits.

Additionally a link could be added to the website to start the persuasive system. The system will mainly depend 
on other advertisers' statistics with the aim of showing how their businesses developed and their profits increased from putting advertisement on this website. Therefore, the system should allow current advertisers to share their experiences and explain the benefits they gained from advertising on that website. This could be done by adding numbers and statistics regarding the improvement they found on their profits taking into account the advertising fees to reveal if they were satisfied or not. Several methods could be exercised in order to encourage advertisers to constantly add their data to the system like; giving discounts on the total advertising costs, or giving higher priority for their advertisement in the system so it will appear more often on the customers' pages.

The system's major work is to collect the numbers and statistics added by advertisers and process these data entries to generate persuading procedures that could be used to convince other business owners to advertise on the website. Mainly, this process will depend on showing new advertisers how other businesses improved and gained more profit by advertising on this web site, using numbers and real data, in order to convince them that they can also enhance their income. The system may also show messages from all advertisers explaining their experiences using web advertising, and may also allow new advertisers to contact them for further information; however this depends on the approval of advertisers in advance.

\subsection{Best Tourism Experience}

Most tourists, who plan to visit a particular city, search the internet prior to their travel to get an insightful view and gain helpful information about their requirements. Like the places they should see, the activities they should do, hotel reservations, maps, transportation, restaurants, etc. The problem is that there is no real user experience in the internet to utilize this, rather all the information available are from private companies showing only one side of the story. And the small chunks of data available from users are distributed on forums and appear as comments on articles and news.

In this system residents of a specific city can share their familiarity and knowledge about the aspects of their city to benefit others. This may help visitors to live a great experience when visiting the city, and will also persuade more people to visit it on the long term. Tourists can also participate by sharing their experience after visiting this city. Not only will this data be useful for the residents and for visitors, it also will be useful for the private sector. A restaurant or a hotel for example will have a feedback from large number of users, and will use this data to improve its service. This will have great effects on the quality of life in that city on the long term.

The data in this system especially for the service sector should be calculated on a monthly basis with the ability to show the overall evaluation. As this is not constant and may change by time. New data for places and activities may be very important but still does not have the same number of recommendations from users as old data. So we can depend on the percentage of recommendations per month instead of the total.

In the example shown in Figure 4, the user has chosen three hotels to compare, depending on some pre-specified criteria like the number of stars, or his budget, or hotel location, etc. The system will give the user a graph that shows other users opinions about different aspects in these hotels for the last month. Then the user can decide which hotel with the best characteristics that suit him, instead of choosing randomly.

\section{Conclusion}

A new dimension of the internet scope is to be built which depends on users as the main source of data, not as receivers of information only. Everyone has his own experience in life that can be useful to others, if it is collected and analyzed correctly. Because this is the age of information revolution, even the smallest piece of information matters. Based on these thoughts we suggested implementing a user experience based persuasive system which depends on building persuasive system using the collected knowledge from users.

Many websites available nowadays provide methods for collecting and managing users' feedbacks, but most of them represent this data using the popular five stars ranking method, which does not give useful detailed information for users. In most cases, a link is provided for users to view more details about the ranking, and to read users' comments, but getting the required information is usually complicated and time consuming. Our work aimed to make the users feedback more readable, useful and significant. The data is displayed in more meaningful methods, such as charts and tables, providing a great help for customers in decision making process.

Three examples have been shown throughout this paper where various systems could be employed. The first example showed how this system can be very useful in E-commerce and E-auction, where it will provide the user with information from real users, rather than having information from the manufacturer or experts only. The second example was about improving web sites revenues from advertisements, and how to improve the web marketing sector. While the last example viewed how this system can help in creating a great tourism experience by using users feedback. 
The same idea represented in those examples could be implemented in almost any e-service available. Private universities can use persuasive systems using previous students input, in order to convince new students to join their university. Job search websites can also persuade companies to supply them with job vacancies and can also persuade job seekers to add and update their data. The same suggestion goes for hospitals, banks, insurance companies, etc.

\section{References}

Alison, P. (2009). Persuasive Decision Support: Improving Reliance on Decision Support Systems. Proc. of Pacific Asia Conference on Information Systems (PACIS 2009), Hyderabad, India: Association of Information Systems. [Online] Available: http://aisel.aisnet.org/pacis2009/11.

Andrews P., Manandhar, S., \& De Boni, M. (2008). Argumentative Human Computer Dialogue for Automated Persuasion. Proc. of the 9th SIGdial Workshop on Discourse and Dialogue, pp. 138-147. [Online] Available: http://portal.acm.org/citation.cfm?id=1622093.

Arroyo, E., Bonanni, L., \& Selker, T. (2005). Exploring feedback and persuasive techniques at the sink. Proc. of the SIGCHI Conference on Human Factors in Computing Systems (CHI 2005), pp. 631-639. doi: 10.1.1.94.7877. Cheng, R. (2003). Persuasion Strategies for Computers as Persuasive Technologies. Department of Computer Science, University of Saskatchewan. doi; 10.1.1.119.7064.

Chittaro L. \& Zangrando N. (2010). The Persuasive Power of Virtual Reality: Effects of Simulated Human Distress on Attitudes towards Fire Safety. Proc. of PERSUASIVE 2010: 5th International Conference on Persuasive Technologies, LNCS 6137, Springer, Berlin, pp. 58-69. doi: 10.1007/978-3-642-13226-1_8.

Consolvo, S., Landay, J. A., \& McDonald, D. W. (2009). Designing for Behavior Change in Everyday Life, IEEE Computer Society. doi:10.1109/MC.2009.185.

Cosley, D. Lam, S. K., Albert, I., Konstan, J. A., \& Riedl, J. (2003). Is Seeing Believing? How Recommender Interfaces Affect Users' Opinions. Proceedings of the conference on Human factors in computing systems, pp. 585-592. doi: 10.1.1.2.6430.

DiMicco, J. M., Pandolfo, A., \& Bender, W. (2004). Influencing Group Participation with a Shared Display. Proc. of Computer Supported Cooperative Work (CSCW'04). ACM, Chicago, Illinois, USA, pp. 614-623. doi $=10.1 .1 .93 .545$.

Fogg, B. J. (1998). Persuasive computers: Perspectives and research directions. Proc. of the SIGCHI Conference on Human Factors in Computing Systems (CHI 1998), pp. 225-232. doi: 10.1.1.83.7257.

Fogg, B. J. (1999). Persuasive Technologies. Communications of the ACM, 42 (5): 26-29. doi: 10.1.1.119.7064

Fogg, B. J. (2003). Persuasive Technology: Using Computers to Change What We Think and Do. Morgan Kaufmann Publishers, San Francisco. ISBN-13: 978-1-55860-643- 2.

Fogg, B. J. (2009). A Behavioral Model for Persuasive Design. Proc. of Persuasive'09, California, USA. doi: $10.1145 / 1541948.1541999$.

Grasso, F., Cawsey, A., \& Jones, R. (2000). Dialectical argumentation to solve conflicts in advice giving: a case study in the promotion of healthy nutrition. International Journal of Human-Computer Studies, 53, 1077-1115. doi: 10.1.1.24.1870.

Herlocker, J. L., Konstan, J. A.. \& Riedl, J. P. (2000). Explaining collaborative filtering recommendations. Proc. of the 2000 ACM conference on Computer supported cooperative work, pp. 241-250. doi: 10.1.1.26.5594.

Intille, S. S. (2004). Ubiquitous computing technology for just-in-time motivation of behavior change. Proc. of 11th Word Congress on Medical Informatics (Medinfo-2004), pp. 1434-1437. doi: 10.1.1.5.7011.

Intille, S. S., Kukla, C., Farzanfar, R., \& Bakr, W. (2003). Just-in-Time Technology to Encourage Incremental, Dietary Behavior Change. Proc. of the AMIA 2003 Symposium. doi:10.1.1.15.1456. 
Johnson, E. M., Koh, H., McAtee, J., \& Shoulders, S. (2007). Persuasive technology to promote conscious driving habits. CHI '07 Extended Abstracts on Human Factors in Computing Systems. [Online] Available: http://www.jamiemcatee.com/portfolio/papers/CHI07Submission.pdf.

Khaled, R. (2008). Culturally-Relevant Persuasive Technology. PhD thesis. Victoria Univ. of Wellington. [Online] Available: http://researcharchive.vuw.ac.nz/bitstream/handle/10063/365/thesis.pdf?sequence=1.

Khaled, R., Biddle, R., Noble, J., Barr, P., \& Fischer, R. (2006). Persuasive Interaction for Collectivist Cultures. Proc. of the Seventh Australasian User Interface Conference. doi: 10.1.1.60.4491.

King, P. \& Tester, J. (1999). The Landscape of Persuasive Technologies, communications of the ACM, 42(5): 31-38. doi: $10.1145 / 301353.301398$.

Konstan, J. A. (2004). Introduction to Recommender Systems: Algorithms and Evaluation. ACM Transactions on Information Systems, 22 (1): 1-4. doi: 10.1145/963770.963771.

Marcus, A. \& Chen, E. (2002). Designing the PDA of the future. Interactions, 9(1): 34-44. doi: 10.1145/503355.503356.

Mathew, A. P. (2005). Using the environment as an interactive interface to motivate positive behavior change in a subway station. CHI '05 Extended Abstracts on Human Factors in Computing Systems, pp. 1637-1640. doi: $10.1145 / 1056808.1056985$.

Meschtscherjakov, A., Wilfinger, D., Scherndl, T., \& Tscheligi, M. (2009). Acceptance of Future Persuasive In-Car Interfaces Towards a More Economic Driving Behavior. Proc. of First International Conference on Automotive User Interfaces and Interactive Vehicular Applications (AutomotiveUI'09), Essen, Germany, pp. 115-118. doi: 10.1145/1620509.1620526.

Morris, M. E. (2005). Social Networks as Health Feedback Displays. IEEE Internet Computing, 9: 29-3. doi: 10.1109/MIC.2005.109.

Nguyen, H. \& Masthoff, J. (2010). Designing persuasive dialogue systems: Using argumentation with care. Lecture Notes in Computer Science, Vol. 5033, Springer, pp. 201-212. doi: 10.1.1.157.9144.

Oinas-Kukkonen, H. \& Harjumaa, M.: Towards Deeper Understanding of Persuasion in Software and Information Systems. Proc. of the 1s International Conference on Advances in Computer-Human Interaction (ACHI.2008), pp. 200-205 (2008). doi: 10.1109/ACHI.2008.31.

Ramos, C., Augusto, J., \& Shapiro, D. (2008). Ambient Intelligence - The Next Step for Artificial Intelligence. IEEE Intelligent Systems Special Issue on Ambient Intelligence, 23 (2): 15-18. doi: 10.1109/MIS.2008.19.

Tester, J., Fogg, B. J., \& Maile, M. (2000). A prototype of persuasive in-car entertainment. CHI'00 Extended Abstracts on Human Factors in Computing Systems, ACM Press, The Hague, pp. 24-25. doi: 10.1145/633292.633309.

Tomlinson, B. (2008). Prototyping a community-generated, mobile device-enabled database of environmental impact reviews of consumer products. Proc. of the 41st Hawaii International Conference on System Sciences, pp.154. doi: 10.1.1.130.3320.

Vande-Moere, A. V. (2007). Towards Designing Persuasive Ambient Visualization. Proc. of Pervasive'07 Workshop: W9 - Ambient Information Systems. doi:10.1.1.142.8193.

Zhang, J. \& Pu, P. (2006). Performance Evaluation of Consumer Decision Support Systems. International Journal of E-Business Research, 2(3): 28-45. doi: 10.1.1.70.7163. 
Table 1. Ranking according to users' feedback

\begin{tabular}{|l|c|c|c|c|c|c|}
\hline \multicolumn{1}{|c|}{ Mobile } & $\begin{array}{c}\text { SW } \\
\text { problems }\end{array}$ & $\begin{array}{c}\text { Network } \\
\text { strength }\end{array}$ & $\begin{array}{c}\text { Battery } \\
\text { life time }\end{array}$ & $\begin{array}{c}\text { Battery } \\
\text { usage time }\end{array}$ & Durability & Overall \\
\hline HTC HD2 & $11 \%$ & $93 \%$ & $85 \%$ & $82 \%$ & $92 \%$ & $88.2 \%$ \\
\hline $\begin{array}{l}\text { Samsung } \\
\text { Mesmerize i500 }\end{array}$ & $22 \%$ & $85 \%$ & $86 \%$ & $84 \%$ & $86 \%$ & $83.8 \%$ \\
\hline $\begin{array}{l}\text { Blackberry } \\
\text { Torch 9800 Slider }\end{array}$ & $19 \%$ & $91 \%$ & $86 \%$ & $82 \%$ & $78 \%$ & $83.6 \%$ \\
\hline Nokia N8 & $17 \%$ & $80 \%$ & $80 \%$ & $88 \%$ & $85 \%$ & $83.2 \%$ \\
\hline Sony Ericsson W8 & $25 \%$ & $88 \%$ & $92 \%$ & $80 \%$ & $81 \%$ & $83.2 \%$ \\
\hline
\end{tabular}

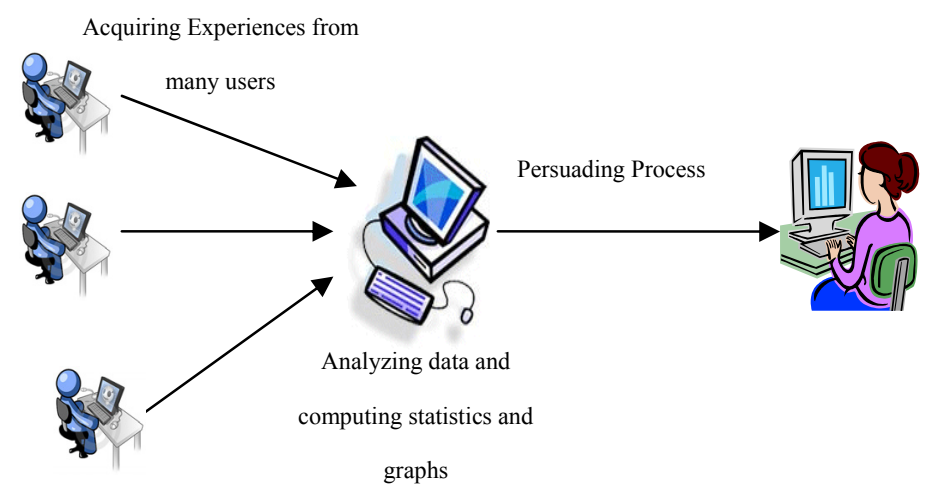

Figure 1. User Experience Based Persuasive System

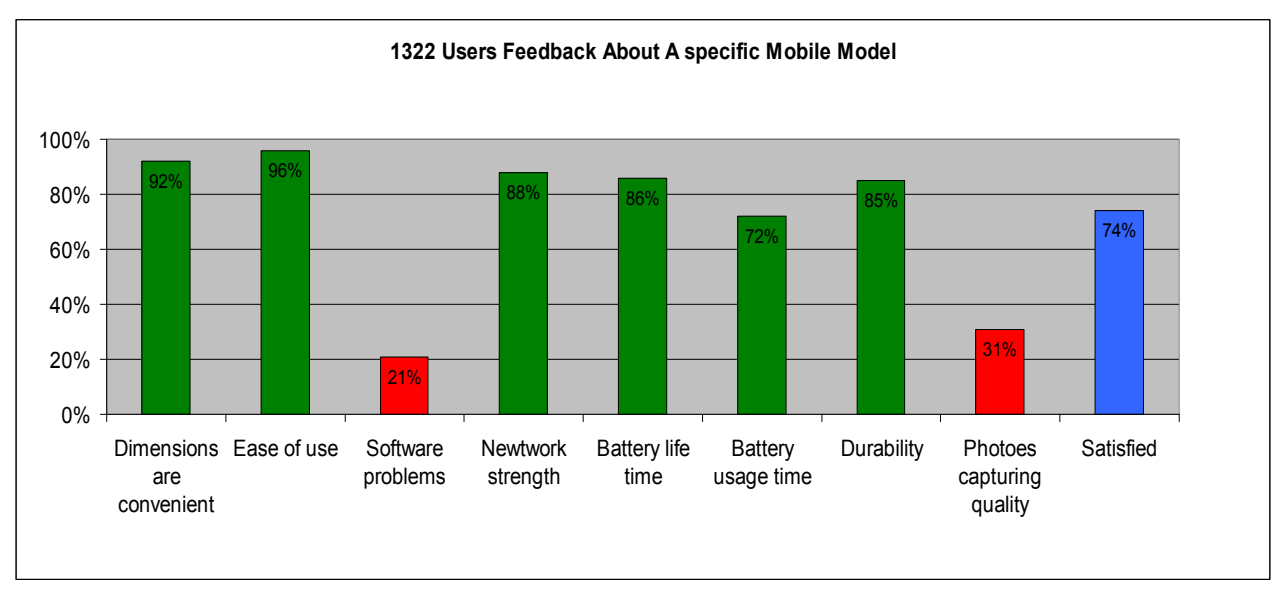

Figure 2. Example 1 of representing users' feedback in the form of a graph 
Please specify your requirements

$\begin{array}{ll}\square \text { 3G } & \square \text { Network Strength } \\ \square \text { Wi-Fi } & \square \text { Battery life time } \\ \square \text { FMRadio } & \square \text { Battery usage time } \\ \square \text { Bluetooth } & \square \text { Software problems } \\ \square \text { Video Support } & \square \text { Durability } \\ \square \text { TV } & \square \text { Ease of use } \\ \square \text { Touch Screen } & \square \text { Dimotoes captured Quality } \\ \square \text { SD Games } & \square \text { Dims are convenient }\end{array}$

Internal Memory more than

Camera Resolution more than

\begin{tabular}{|l|}
\hline $8 \mathrm{MB}$ \\
\hline 3MPixels \\
\hline $150 \mathrm{MS} \$$ \\
\hline
\end{tabular}

Price less than

Submit

Figure 3. Specifying User requirements

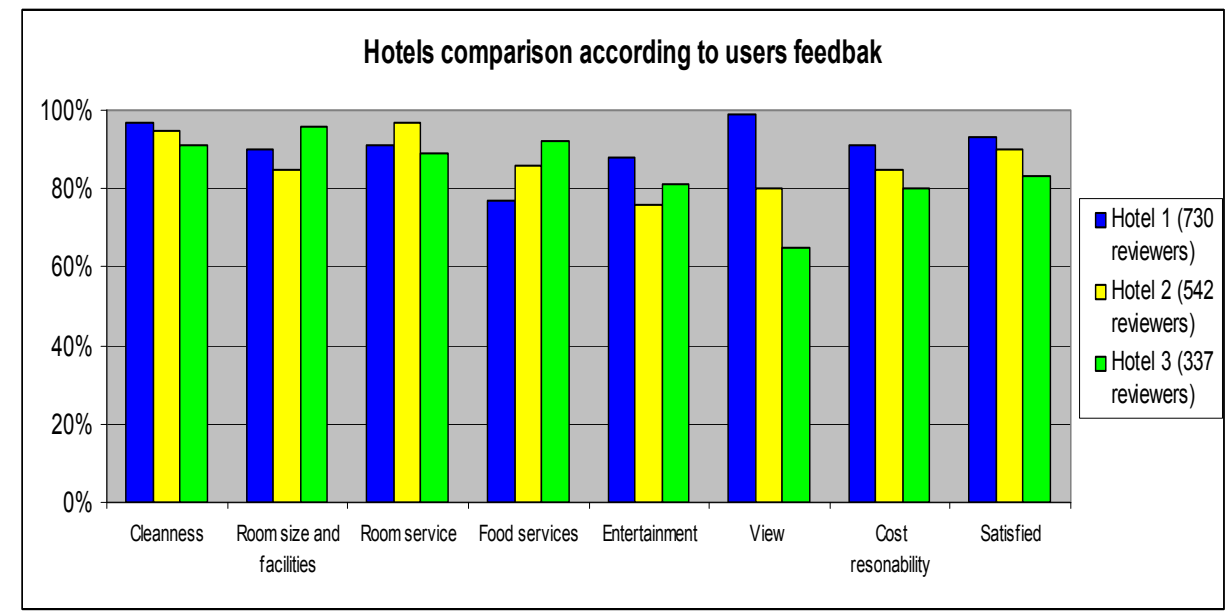

Figure 4. Example 2 of representing users' feedback in the form of a graph 